\title{
UN APORTE A LA EDUCACIÓN COMO PRÁCTICA DE LIBERTAD LA CÁTEDRA GESTIÓN Y DESARROLLO DE LA UIUIENDA POPULAR
}

\author{
Marta GIRÓ; Rafael FRANCO; María B. PELLI; Elizabeth PACE; \\ Mariana CAMPOS; Noel DEPETTRIS; Rosario OLMEDO; Diego PONZIO \\ martagiro45@gmail.com \\ Los autores son el equipo docente actual de la cátedra GDVP. Titular, Arq. Marta \\ Giró. Adjunto Arq. Rafael Franco. Jefa de Trabajos Prácticos Mgt. Arq. María B. Pelli. \\ Auxiliares de primera categoría: Mariana Campos, Noel Depetris. Adscriptos: Rosario \\ Olmedo y Diego Ponzio. Profesora invitada: doctora abogada Elizabeth Pace.
}

\section{PALABRAS CLAUE \\ Educación; hábitat social; problemática habitacional.}

\section{RESUMEN}

La intención de esta ponencia es presentar una propuesta formativa, precursora, innovadoray motivadora para la creación de otras cátedras en distintas universidades de nuestro país y de Latinoamérica, a treinta años de su creación. Intentaremos recuperar brevemente el camino recorrido a través de algunos ejemplos de prácticas de enseñanza-aprendizaje, llevados adelante durante los últimos años, en las dimensiones docencia, extensión e investigación. También presentaremos una nueva propuesta curricular como continuidad del camino iniciado. Este complejo académico pretende consolidar una modalidad de acción que articule la teoría con la práctica concreta, cogestionando con otras disciplinas, otros actores sociales y, fundamentalmente, con los destinatarios.

\section{KEYWORDS}

Education; Social habitat; Housing problem

\section{ABSTRACT}

The intention of this paper is to present a formative, pioneering, innovative and motivational proposal for the creation of other chairs in different universities in our country and in Latin America, thirty years after its creation. We will try to briefly recapitulate the way forward through some examples of teaching-learning practices, carried out during the last years, in the teaching, extension and research dimensions. We will also present a new curricular proposal as a continuity of the path started. This academic complex aims to consolidate a modality of action that articulates the theory with the concrete practice, comanaging with other disciplines, other social actors and, fundamentally, with the recipients.entalmente, con los destinatarios. 


\section{LA EXPERIENCIA}

\section{Historia de logros, obstáculos y desafíos}

La actual cátedra de Gestión y Desarrollo de la Vivienda Popular (GDVP) de la carrera de Arquitectura de la facultad de Arquitectura y Urbanismo de la Universidad Nacional del Nordeste (FAU-UNNE) de Resistencia, Chaco, se gestó como un nuevo paso del proyecto general de abordaje del problema de la vivienda social, que se puso en marcha en 1967, con la creación del Departamento de Diseño Arquitectónico, en la misma facultad, por iniciativa del arquitecto Víctor Saúl Pelli, quien fue su director hasta marzo de 2011. Al ir consolidándose la acción con las prácticas de extensión y la estructura conceptual con la investigación, en los primeros años de la década del 70, empezó a tomar forma la idea de ampliar el proyecto con la práctica docente, como otra manera de explorar, elaborar y multiplicar conocimientos sobre la vivienda social. Si bien esta iniciativa no encontraba en ese momento obstáculos institucionales de peso, su concreción se vio largamente demorada, primero por la desdichada etapa del gobierno de facto en la Argentina entre 1976 y 1983 y, luego, por las turbulencias propias del proceso de reestructura- ción de la Universidad en los años de recuperación del estado de derecho.

La propuesta de una cátedra dedicada a la problemática social de la carencia habitacional en una facultad de Arquitectura, localizada en una de las provincias más pobres de la Argentina, era bien vista por las nuevas autoridades y celebrada por el movimiento estudiantil en sus diferentes agrupaciones, pero el clima institucional y académico presentaba otras prioridades, por lo que la iniciativa recién se pudo concretar en el año 1987, como una asignatura electiva de la carrera de Arquitectura, veinte años después de la creación del Departamento de Diseño Arquitectónico. Como refieren los conceptos de Freire y VieIRA Pintos (Freire, 2014: 113), el "acto límite" sobrepasó la "situación límite" o "margen real donde empiezan todas las posibilidades" y permitió, de esa manera, superar la frontera del "ser" para llegar al "ser más".

La asignatura se llamó, inicialmente, Introducción a la Vivienda Económica, nombre que buscaba adecuarse a la sensibilidad de esos tiempos de transición. No había antecedentes de una asignatura que tuviera como objeto de estudio la pobreza habitacional urbana en las facultades de arquitectura argentinas, y tampoco el equipo docente a cargo de la cátedra contaba con experiencia en

1. FreIRE Cita a VIEIRA PINTO en La Pedagogía del Oprimido: "Para Vieira Pinto, las 'situaciones límites' no son 'el contorno infranqueable donde terminan las posibilidades, sino el margen real donde empiezan todas las posibilidades'; no son 'la frontera entre el ser y la nada, sino la frontera entre el ser y el ser más (más ser)'. Alvaro Vieira Pinto, Consciencia e realidade nacional, Iseb, Río, 1900, vol. II, p. 284."

2. A fines de la década de los 90, el Departamento de Diseño Arquitec tónico comenzó a llamarse Instituto de Investigación y Desarrollo en Vivienda (IIDVi), denominación que conserva hasta la actualidad.

la organización de una materia que en las facultades de Arquitectura se denomina "teórica" al no ser un taller de proyecto. Sin embargo, el equipo docente inicial contaba con una amplia experiencia educativa en los talleres de diseño, que resultó valiosa como punto de partida de la nueva asignatura. Los modelos de organización de las unidades académicas correspondientes a otras subáreas de la arquitectura no se adaptaban a lo que se buscaba crear con esta cátedra, por lo que la asignatura debía construirse desde cero. Los contenidos explorados en el Departamento de Diseño Arquitectónico, la estructura general del curso y los métodos pedagógicos debieron ser congruentes entre sí $y$, además, pertinentes con el perfil de los alumnos, que cambiaba año tras año. Este fue uno de los mayores riesgos, el de no mantener la coherencia entre el discurso y la práctica, entre el "decir" y el hacer.

En el primer año, la asignatura tuvo diez alumnos, pocos en relación con la escala de la facultad, pero se trataba de un grupo de excelente nivel, que eligieron ser parte de esta aventura formativa. Y lo fueron. Se acordó de entrada que ayudarían al proceso de otorgar a la materia una forma académica adecuada para interrelacionarse con los conocimientosque se estaban elaborando en el Departamento de Diseño Arquitectónico ${ }^{2}$. También se acordó definir en forma conjunta los modos más adecuados de relación entre contenidos, docentes y alumnos. Además, se estableció la reunión semanal de cátedra para evaluar la clase anterior y definir sobre la base de esa evaluación las siguientes. Esta primera experiencia fue muy 
valiosa para la estructuración pedagógica y para la definición de un método de trabajo caracterizado por una constante formulación, evaluación participativa y consecuente reformulación anual de contenidos, programas y estrategias, siempre manteniendo su esencia: la introducción al aprendizaje de un modelo de intervención sobre una realidad, al momento relegadaporloscontenidos universitarios, para transformarla.

En 1994 se decidió pedir a la facultad el cambio de nombre de la asignatura, y se propuso uno que expresara con mayor propiedad su naturaleza y su base conceptual. El actual nombre, Gestión y Desarrollo de la Vivienda Popular, fue aprobado en 1996. Se buscaba con este nombre, por un lado, recortar y delimitar el amplio campo de interés de la vivienda humana al campo a la "vivienda social" $y$, por el otro, definir su naturaleza centrada en el conocimiento a fondo del problema y de las soluciones a través del desarrollo de procesos regidos por modelos de gestión participativos.

\section{Conocer el mundo para intervenir en él. La estructura del curso}

Enestecontexto, elcursoseestructuró en tres partes no necesariamente correlativas. La primera se destinó al conocimiento y comprensión de la problemática a partir de una "inmersión" en una situación de precariedad habitacional real. Esta parte también se reservó para la objetivación de los conocimientos previos y las representaciones de los estudiantes sobre la temática, aspectos fundamentales para lograr, siguiendo a Ausubel (CARretero, 1993: 27), "aprendizajes significativos". La segunda parte se centró en conocer e internalizar los instrumentos conceptuales y técnicos para abordar y resolver la problemática en forma integral. La última parte se dedicó a poner en acto esos instrumentos, con la elaboración de un proyecto de gestión integral y participativa que generase vías de solución a la situación de precariedad habitacional estudiada. Esta estructuración, que se mantiene en la actualidad, permitió que la experiencia pedagógica avanzara, creciese y también que se transformara de acuerdo con las exigencias de los nuevos contextos educativos.

\section{Lograr el deseo de aprender. "El nuevo desafío": el paso de materia electiva a asignatura obligatoria en la FAU}

En el año 2008 la asignatura fue incorporada al currículo de grado de la facultad, con carácter de cursado obligatorio, y transformó así la situación casi marginal que se le daba a esta temática con el carácter de electiva en el ámbito institucional de nuestras facultades de arquitectura. Esta jerarquización, si bien constituyó un logro largamente deseado, enfrentó al equipo docente con dos tipos de dificultades: los problemas operativos y la resistencia que el paradigma disciplinar tradiciona ofrecíaalaincorporación de temáticas relacionadas con la pobreza habitacional en el currículo de arquitectura. Los problemas operativos, que eran comunes al resto de las asignaturas obligatorias, como la insuficiencia de cargos docentes para enfrentar dificultades propias de la masividad (se pasó de tener treinta a cincuenta estudiantes por docentes aproximadamente), losespaciosfísicos inadecuados para la modalidad de trabajo delcurso, los obstáculos burocráticos y prácticos para la implementación de trabajos de campo, entre otros aspectos, dificultaron la implementación de estrategias didácticas pensadas para la situación anterior.

Lo segundo, la resistencia que ofrecía el paradigma disciplinar dominante al cambio, era específico de nuestra asignatura por la temática abordada. En los hechos se verificaba, por un lado, en las dificultades del trabajo docente con alumnos con un fuerte imaginario disciplinar que provocaba conflictos y reticencias en la comprensión y reflexión sobre los crecientes desafíos que plantea la cuestión habitacional en Latinoamérica en general y en el NEA en particular; por otro lado, en la rigidez estatutaria y administrativa de nuestra universidad paragenerar los procesos interdisciplinarios de enseñanzaaprendizaje tendientes a afrontar la complejidad que implica la problemática que abordaba la asignatura.

Esta nueva situación requirió por parte del equipo docente afrontar el desafío de pasar de una situación particular - y podríamos decir "privilegiada" en relación con las condiciones y características con las que desarrollaba el proceso de enseñanza aprendizaje (treinta a cuarenta estudiantes, que elegían cursar la asignatura) - a una realidad que exigía rediseñar estrategias pedagógicas adecuadas, tendientes 
a dar respuesta a los obstáculos y desafíos que presentaba la nueva situación curricular. En este contexto, se decidió conservar algunas características de la situación anterior e innovar en otras. Lo que se mantuvo fue lo siguiente: a) el programa oficial de la asignatura que definía el objeto de estudio, los objetivos pedagógicos y los principales contenidos; $b$ ) la concepción de la evaluación que valorizaba los procesos sobre los resultados; $c$ ) la interacción con el medio, que consistía básicamenteen conocer una situación de pobreza habitacional concreta a través de convenios de articulación con instituciones públicas y privadas y $d$ ) las reuniones semanales de cátedra de evaluación y preparación de clases. Lo que se decidió variar fueron las estrategias pedagógicas, el carácter de las entregas de los trabajos prácticos parciales y finales y las comunicaciones con los alumnos, incluyendo los medios digitales. También se amplió el número de docentes y se solicitaron nuevos cargos y adscripciones de alumnos avanzados. En esta dirección, y para encuadrar la cátedra en el régimen curricular obligatorio, se instrumentó una modalidad de entrega de trabajos prácticos, con carácter de exámenes parciales, y para los alumnos que no alcanzaran a cumplir con los objetivos de la asignatura, se planteó una instancia de coloquio final, consistente en una reunión donde los alumnos explicitarían su proceso de aprendizaje, identificando obstáculos y logros. El cambio produjo la conmoción que todo cambio de este tipo provoca. Se interrumpió una continuidad, pero las previsiones implícitas y explícitas tomadas por el grupo durante la preparación del curso hicieron posible enfrentar las demandas propias del nuevo escenario, al proponer estrategias dinámicas, fácilmente adaptables en relación con las secuencias de contenidos, centradas fundamentalmente en la integración grupal al proceso de aprendizaje.

\section{Modulando el ritmo y adaptando los dispositivos}

Finalizado el año 2008 y hasta 2011 , sedecidióabordar la problemática de las ocupaciones masivas de tierras ocurridas entre fines del año 2007 y principios de 2008 en la ciudad de Resistencia, Chaco, Argentina, que involucra una diversidad de cuestiones que actúan interdependientemente y constituyen la estructura de un sistema que funciona como una totalidad organizada o sistema complejo (GARcía, 2007: 21). A partir del reconocimiento explícito por parte del equipo docente de la asignatura de que una problemática compleja está determinada por la confluencia de múltiples factores que interactúan entre sí pero que "no pueden ser estudiados aisladamente", y que no puede ser descrita, explicada ni resuelta desde el enfoque de profesionales de una disciplina ni "sumando" simplemente enfoques parciales que la aborden en forma independiente, se advirtió la demanda de incorporar docentes de otras disciplinas para estudiary proponer vías de solución posibles a la cuestión multidimensional que planteaba la materia. La misma demanda fue detectada en las evaluaciones al curso realizadas por los estudiantes al finalizar cada año.

La interdisciplinariedad en la ense- ñanza representaba para el equipo docente una necesidad largamente esperada desde la creación de la asignatura, e implicaba la desnaturalización de las fuerzas conservadoras del paradigma disciplinar vigente existentes, presentes incluso en los discursos y prácticas de los mismos docentes de la asignatura. El cambio que se propuso no solamente se centraba en cuestiones disciplinarias, sino que abarcaba aspectos epistémicos, pedagógicos y políticos

\section{Construcción de un espacio interdisciplinar}

En este marco se inició un proceso gradual, tendiente a enmarcar institucionalmente la participación e integración de profesionales y docentes de distintas disciplinas, destinado a estimular voluntades, viabilizar compromisos e iniciar un proceso de formación académica interdisciplinaria para el abordaje de la problemática de la gestión social del hábitat. Los logros en este sentido fueron los acuerdos de cooperación celebrados entre las facultades de Arquitectura y Urbanismo, de Derecho y el Instituto de Ciencias Sociales. En este marco normativo, se integró una profesional de la abogacía y una profesional de trabajo social. En una instancia posterior, se incorporó una profesional de la facultad de Ciencias Económicas El espacio interdisciplinar docente se fue consolidando y ampliando a partir de la toma de contacto y la firma de un acuerdo de cooperación con el Instituto Superior de Servicio Social Remedios de 
Ministerio de Planificación Federal Inversión Pública y Servicios de la Nación

Ilustración 1. Articulación Intersectorial. Intervención en asentamiento Soberanía.

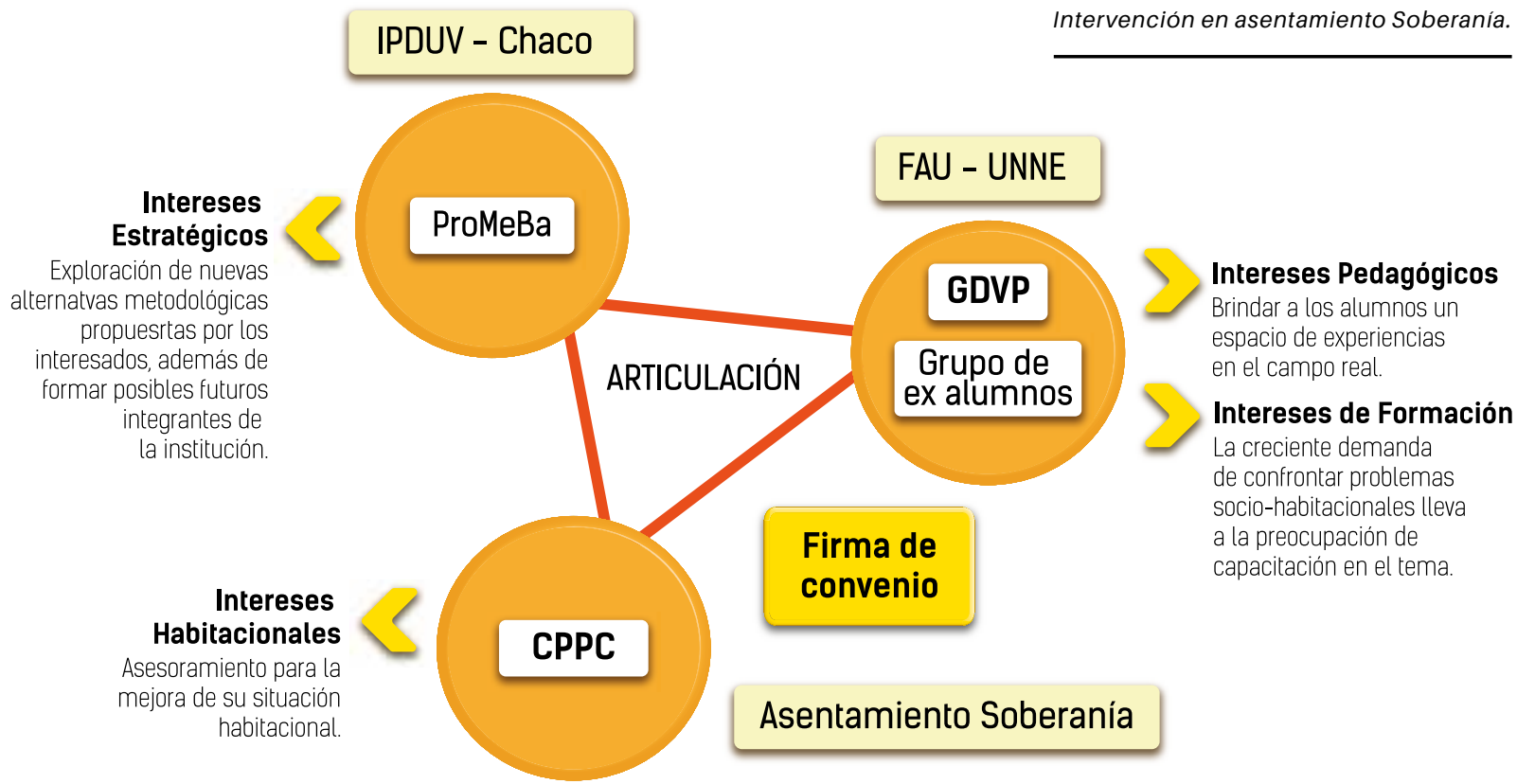

Escalada de San Martín, por el cual se incorporaron al equipo docente estudiantes avanzados de trabajo social, en carácter de adscriptos.

Se inauguraba así esta nueva experiencia con fuertes expectativas con respecto a los nuevos integrantes y a los cambios que esto significaba para la asignatura. Para llevarla adelante, se consensuaron un marco epistémico y una metodología pedagógica para abordar el objeto de estudio. Como resultante de esta acción, se produjo la asimilación del objeto a estos esquemas teóricos, lo que permitió, en un proceso conjunto con los alumnos, organizar lo recogido de la trama real, recrear el objeto, estudiar su carácter multidimensional, comprender las razones y explicar los procesos a partir de relaciones y, a partir de esto, derivar acciones para su solución. Esta idea, surgida de intuiciones y certezas, se fue transformando primero en un proyecto y después en un plan de acción. Para que esta transformación fuera posible, fue necesario hacerla operativa a través de la definición de objetivos y metas que señalaran una dirección y un trayecto que se constituyó en un verdadero aporte a la formación integral del profesional que le permitiera en forma introductoria "saberysaber hacer", conjuntamente con otros profesionales en el campo del hábitat social.

\section{La práctica profesional asistida. Docencia y extensión}

Esto facilitó, entre otras cosas, llevar adelante experiencias concretas de campo y sumar alum nos y docentes, con lo que se profundizó lo realizado hasta ese momento en el camino hacia el abordaje interdisciplinario de situaciones problemáticas complejas.
El equipo docente se propuso en el año 2010 celebrar un acuerdo de cooperación entre estudiantes, docentes de la cátedra GDVP y el Centro de Promoción Comunitaria (CPC), organización territorial no gubernamental que viene trabajando en la zona sur de Resistencia desde hace varios años.

En este marco, y a partir de la solicitud de los dirigentes del CPC, se conformó un grupo inicial de veinte alumnos (10\% del total de los que cursaron la asignatura) para realizar el rediseño del loteo del Asentamiento Soberanía, donde habitaban 120 familias. Para llevar adelante esta tarea, se propuso para la segunda mitad del año encuadrar la labor docente en el Programa de Práctica Profesional Asistida y así ampliar el Acuerdo FAU-Centro de Promoción Comunitaria incorporando al Instituto Provincial de Desarrollo Urbano y Vivienda 
(IPDUV) a través del Programa de Mejoramiento Barrial (Promeba). Esta acción - que obtuvo el reconocimiento institucional de parte de la facultad de Arquitectura con una Declaración de Interés como Tarea Extensionista y una Mención Especial en el Premio ARQUISUR 2015 - brindó a los estudiantes que habian terminado el cursado regular y continuaban interesados en la temática la posibilidad de realizar una experiencia profesional efectiva en el campo del hábitat social.

Al año siguiente, a solicitud del Gobierno de la Provincia de Corrientes, se realizó el diagnóstico para la aplicación del Programa de Mejoramiento Barrial (Promeba), basada en intereses académicos de formación. En el segundo cuatrimestre se celebraron los convenios de cooperación correspondientes entre la cátedra a través de la FAU y

Fuente: equipo de trabajo asenta miento Soberanía
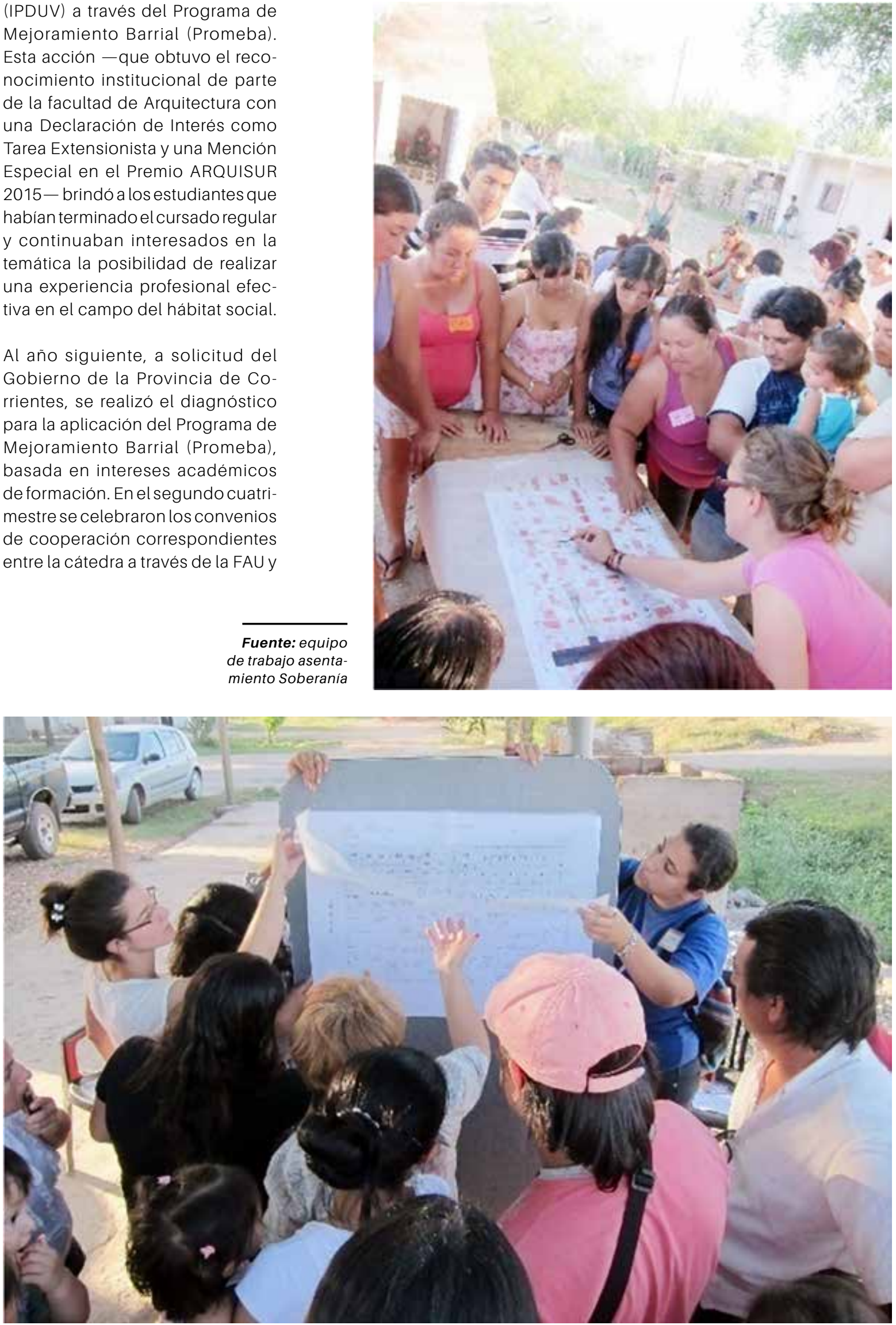
la Unidad Central de Administración y Programación Financiera Internacional (UCAPFI), en el marco del Programa Universidad en el Medio de la UNNE, que financió los insumos para la realización de experiencias de extensión universitaria y permitió continuar poniendo en acto los conocimientos teórico-prácticos adquiridos durante el cursado de la asignatura, profundizando así el proceso deaprendizajeenunespacio no curricular asistido.

El trabajo tenía como objetivo el reordenamiento de las parcelas de familias asentadas en las manzanas Números12, 13, 14, 15y 16 del asentamiento informal La Olla, como un aporte al Proyecto de Inserción Urbana de La Olla, Corrientes, realizado por el UCAPFI para el Promeba. Para alcanzar ese objetivo, se consensuó entre el equipo de la cátedra GDVP (constituida por docentes y estudiantes de arquitectura, abogacia y trabajo social) y los profesionales de la UCAPFI llevar adelante procesos genuinos detransformación social perdurables, para lo cual se adoptó una estrategia de trabajo participativa que articulara actores, intereses y recursos. La articulación de los actores sociales nombrados permitió concretar en el plazo de un año una experiencia interdisciplinaria, interinstitucional e intersectorial, basada en criterios participativos.

Participaron de esta experiencia el Programa Universidad en el Medio de la UNNE; la facultad de Arquitectura y Urbanismo, UNNE, cátedra Gestión y Desarrollo de la Vivienda Popular; habitantes del asentamiento La Olla; la Unidad Central de Administración y Programación Financiera Internacional (UCAPFI), del Gobierno de la Provincia de Corrientes, Argentina; el Programa de Mejoramiento de Barrios (Promeba); la facultad de Derecho y Ciencias Sociales de la UNNE (Acuerdo de Cooperación FAU-FDCS); el Instituto Superior de Servicio Social Remedios de Escalada de San Martín (Acuerdo de Cooperación FAU-ISSRE de SM).

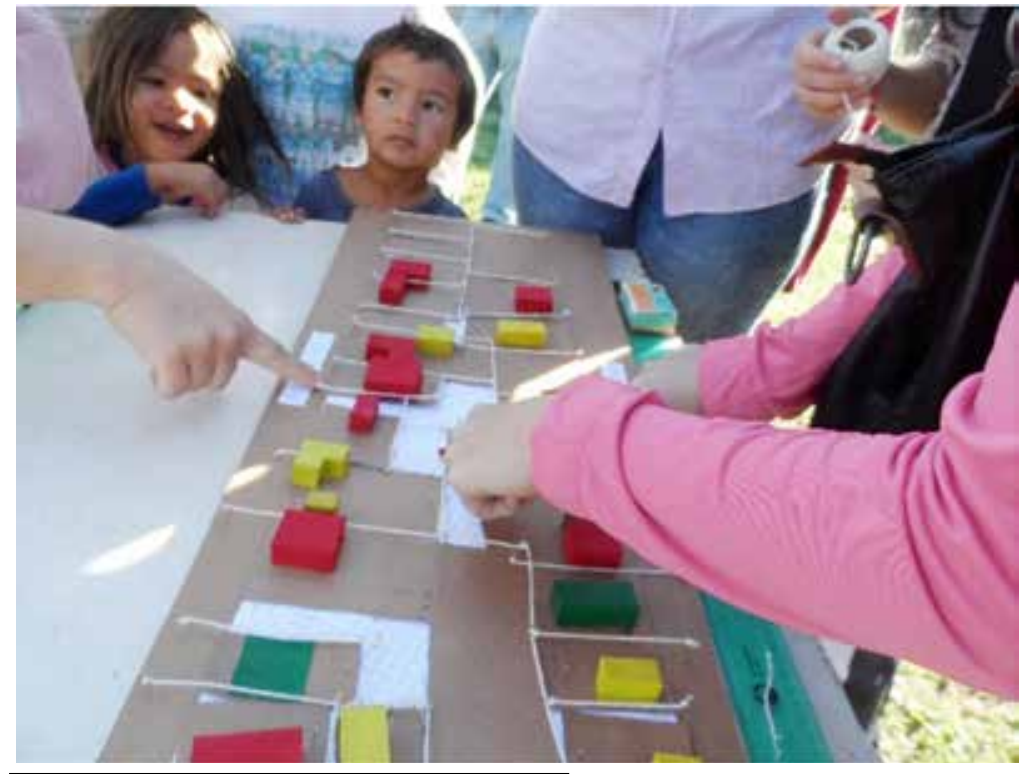

Fuente: equipo de trabajo asentamiento La Olla

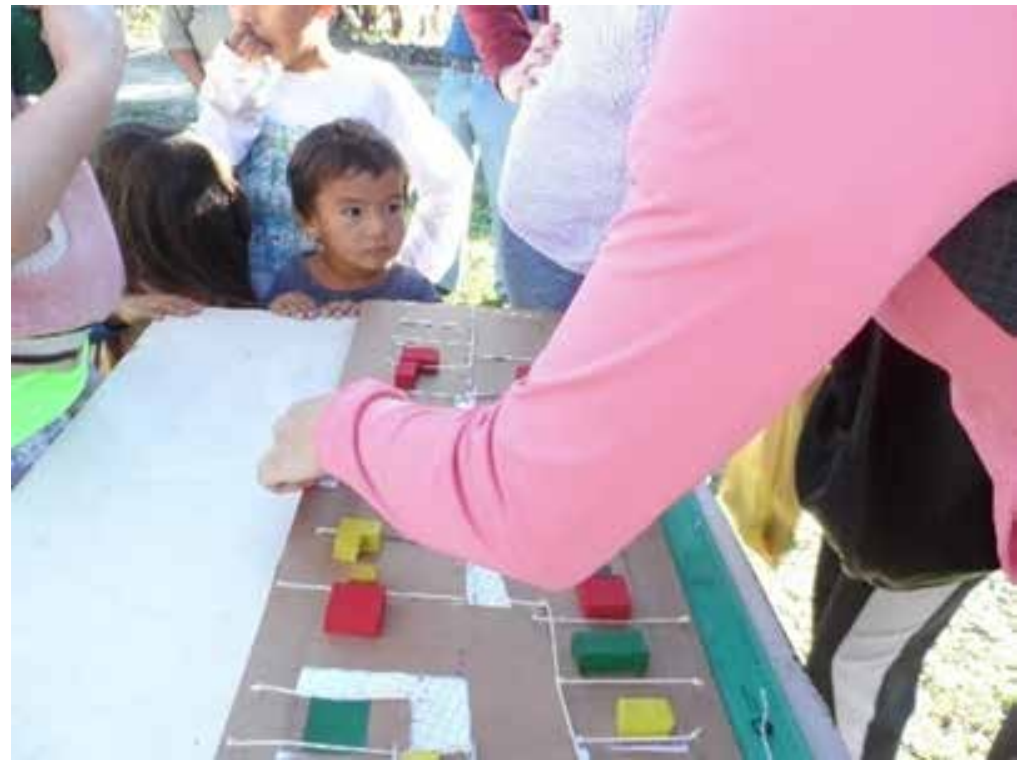


La construcción realizada como cátedra integrante de la RED ULA$\mathrm{CAV}^{3}$ permitió aunar voluntades e iniciar el camino de investigar para reflexionar y perfeccionar las prácticas docentes. En este contexto, se presentó un proyecto de investigación ante la Secretaría General de Ciencia y Técnica de la UNNE (PI C004/010). Este involucró a cuatro cátedras de la RED 4 . El objetivo fue conocer y tipificar los procesos de aprendizaje para abordar la complejidad implicada en la problemática habitacional de los sectores en situación de pobreza seguidos por cátedras afines a la temática en cuatro universidades de tres provincias del país, Chaco, Santa Fe y Córdoba. A partir de este proyecto y capitalizando aprendizajes conceptuales y metodológicos obtenidos de la ejecución del PI C004/010 y de la actividad docente de los equipos de las cátedras participantes, se presentó y acreditó en la Secretaría General de Ciencia y Técnica de la UNNE un segundo proyecto de investigación tendiente a identificar y tipificar los procedimientos seguidos por tres cátedras de tres facultades de Arquitectura de dos provincias argentinas (Chaco y Córdoba) para trabajar los obstáculos epistemológicos y lograr que el conocimiento de la problemática socio-habitacional de los sectores en situación de pobreza en toda su complejidad pueda ser construido, incorporado, resulte duradero y creíble por parte de los alumnos.

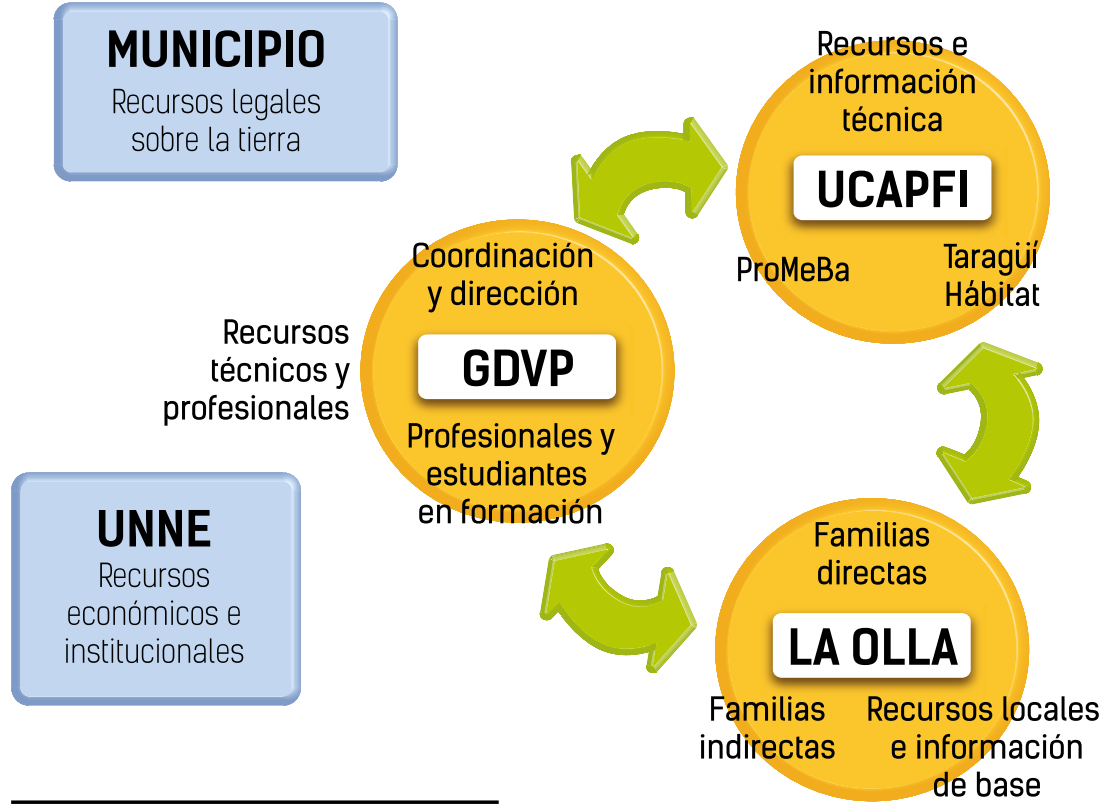

Ilustración 2. Intervención Intersectorial.

Proyecto de Intervención Urbana La Olla, Corrientes.

Articulación entre cátedras de la Red Universitaria Latinoamericana

de cátedras de Vivienda (ULACAV). Investigación en docencia

Entre los principales aprendizajes que recupera este proyecto podemos mencionar:

Que es necesario, según RUMELHARD (CAMILLONI, 1997: 32), reconocer que existen en los alumnos otros tipos de conocimientos al instalar conocimientos nuevos.

Queel paso del conocimiento común al conocimiento científico no se piensa. Es algo que se instala directamente en el saber más actual, sin preocuparse por la posible existencia de otros tipos de conocimientos, y se presenta en el espíritu del alumno como respuesta para tranquilizar

3. La Red ULACAV está integrada por académicos latinoamericanos que abordan en forma permanente la producción social del hábitat de manera interdisciplinaria, a través de actividades de docencia, investigación y extensión, llevadas a cabo desde cátedras de grado y posgrado. La iniciativa surgió en 1994. La red sostiene que el mejor aporte radica en capacitar al máximo en esta problemática a jóvenes generaciones que transitan por la universidad en su trayectoria hacia la adquisición de títulos profesionales (de grado o posgrado). A tal fin orienta su acción hacia el tratamiento formativo, académico, además del aporte de soluciones frente a conflic tos inherentes al hábitat residencial que afectan a la población latinoamericana.

4. Las cuatro cátedras son las siguientes: Problemática de la Vivienda Popular (I y II). FAU-UNC, Córdoba, Argentina; Problemática Socio-habita cional I FAU-UCC, Córdoba; Gestión y Desarrollo de la Vivienda Popular FAU-UNNE, Resistencia, Chaco; Cátedra Interdisciplinaria e Interinstitucional de Vivienda Social ESS, FAU, UNL, UTN, Santa Fe, Argentina.

o para dar sentido, más que para ofrecer una verdadera explicación. Reconocer la necesidad del instalarse en la ruptura con el pensamiento común. El saber científico no tiene solamente la función de responder a preguntas. Tal como lo afirma RUMELHARD, tiene una función polémica, porque reemplaza las creencias que se presentaban como explicaciones verdaderas.

Reconocer que "hacer ciencia es pues abandonar un conocimiento de opinión, un conocimiento mal cuestionado, asertivo, es decir, reducido a una mera comprobación, parallegara un conocimiento que, una vez problematizado, se fundará en la razón. Esto es lo que ocurre en la ruptura fundamental que presenta Bachelard, entre conocimiento común y saber científico". FABRE \& Orange (CAmilloni, 1997, pág. 67).

Examinar los obstáculos para tenerlos en cuenta al diseñar los dispositivosy estrategiasdeenseñanza-aprendizaje. Según Michel Fabrey Christian Orange (CAMILLonı, 1997, pág. 64), para tomar conciencia del cambio conceptual son necesarios dispositivos que contribuyan a la problematización 
y no solo a resolver problemas. El alumno solo puede dar sentido a los conocimientos científicos si los aprendecomosolucionesposibles de problemáticas elaboradas en clase.

De estos aprendizajes se desprende la necesidad de intentar tipologías de procedimientos para superar los obstáculos en el paso del conocimiento común al conocimiento científico, con la única intención de hacer progresar el concepto de obstáculo epistemológico enunciado en 1938 por Gastón Bachelard (BACHELARD, 2000: 15). Los resultados de estos proyectos fueron y son ofrecidos como insumos a instancias de gestión y evaluación de planes curriculares, y aportan a la ampliación de las competencias profesionales y a la interacción entre distintas disciplinas.

\section{Nueva propuesta académica}

Este equipo docente cree posible continuar por el camino de la transformación de los procesos de aprendizaje que conduzcan a lograr un perfil profesional con mayor visión integral y regional. En ese sentido, viene madurando un cambio que seguramente será un nuevo desafío: desarrollar e incorporar al currículo de la FAU otros espacios académicos de abordaje de la problemática que refuercen y amplíen la formación de profesionales consumados y comprometidos con la sociedad.

Una de las propuestas permitirá introducir a los estudiantes en la temática de la producción social del hábitat en el ciclo básico de la carrera (en la ilustración 3 figura con el nombre de Diseño Participativo), paralelamente con los demás contenidos básicos de la carrera de la FAU, para luego con la actual asignatura profundizar el proceso tendiente a ampliar y diversificar la formación profesional, la interdisciplinariedad y la relación teoría-práctica en la formación de profesionales que puedan dar respuestas adecuadas a la complejidad de la problemática socio-habitacional de los sectores en situación de pobreza de nuestra región.

Esta propuesta surge a partir de la certeza de que la mayoría de los alumnos, ya casi profesionales, que comienzan la asignatura cada año, nunca tuvieron contacto con la temática de la pobreza habitacional. Su formación, que en general está orientada a profundizar en los hechos el perfil profesional vigente y a reforzar la división del conocimiento en disciplinas y asignaturas como compartimientos estancos que producen el divorcio entre teoría y práctica, no se adecua al actual contexto profesional, en el cual los organismos encargados de la resolución de la problemática socio-habitacional de los sectores en situación de pobreza requieren con urgencia profesionales adiestrados para articular su actuación con otras dimensiones(social, legal, económica, ambiental etc.), otras escalas como la territorial y con la capacidad para interactuar con profesionales de otras disciplinas y con actores sociales involucrados en la problemática.

Con el convencimiento de la necesidad de crear un fuerte vínculo con la realidad, la cátedra también plantea el desafío de crear otro espacio institucionalizado y permanente, compartido tanto por pobladores como por docentes y estudiantes de distintas disciplinas y distintos actores sociales gubernamentales y no gubernamentales, para poder diseñary ejecutar en forma conjunta acciones concretas de solución a los problemas socio-habitacionales de los sectores en situación de pobreza (en la ilustración 3 figura con el nombre de "La Usina").

Lapresencia, dentrodelaFAU-UNNE, de otra carrera de grado, Diseño Gráfico, de actividad proyectual, con énfasis en la comunicación visual, brinda la oportunidad de introducir la temática del hábitat social también en ella, dándole una nueva mirada a la profesión del diseñador gráfico, como profesional de la comunicación, comprometido con la realidad local y regional, con competencias para desempeñarse en la temática del hábitat. Si bien se han realizado algunos seminarios, charlas y otras iniciativas aisladas, aún no se ha propuesto para esta carrera una introducción sistemática y programada del tema en cuestión.

Los contenidos de la cátedra GDVP también fueron el germen que permitió la creación de la Maestría en Gestión y Desarrollo de la Vivienda Social, que está enmarcada en el Instituto de Investigación y Desarrollo en Vivienda (IIDVi) y conforma el complejo académico en la temática del hábitat social, constituido por docencia de gradoy posgrado, investigación y extensión, los tres campos que le compete desarrollar a la universidad. Esta maestría, que se inició en el año 2013, se articularía con los nuevos espacios propuestos. 
SINTESIS DE LA

\section{PROPUESTA}

(Ver ilustración 3)

1. Crear una cátedra electiva denominada "Diseño Participativo".

Asignatura electiva para Arquitectura y Diseño Gráfico; ubicada entre 2. do y $3 .^{\text {er }}$ año de la carrera de Arquitectura (para ambas carreras). Dictado: primer cuatrimestre.

2. Mantener y fortalecer la interdisciplinariedad de la asignatura Gestióny Desarrollodela Vivienda Popular GDVP.

Asignatura obligatoria para Arquitectura y optativa para Diseño Gráfico; ubicada en $5 .{ }^{\text {to }}$ año de la carrera de Arquitectura. Dictado: primer cuatrimestre.

3. Crear e Institucionalizar un espacio de práctica profesional asistida denominado "La Usina". Asignatura electiva para Arquitectura y Diseño Gráfico. Sería un espacio abierto a la integración de profesionales y estudiantes de otras disciplinas (Comunicación Social, Abogacía, Trabajo Social, Economía, etc.). Ubicada en $5 .{ }^{\text {to }}$ año de Arquitectura y $3 .^{\text {er }}$ y $4 .{ }^{\text {to }}$ años de Diseño Gráfico. Dictado: segundo cuatrimestre.

Dichapropuestatieneaprobacióndel HCD. FAU-UNNE, según Resolución 690 del 24 de noviembre del año 2015. Actualmente se están desarrollando los programas correspondientes a cada asignatura para reencauzar el proceso de negociación con las autoridades de la FAU.
Ilustración 3. Esquema general de la propuesta

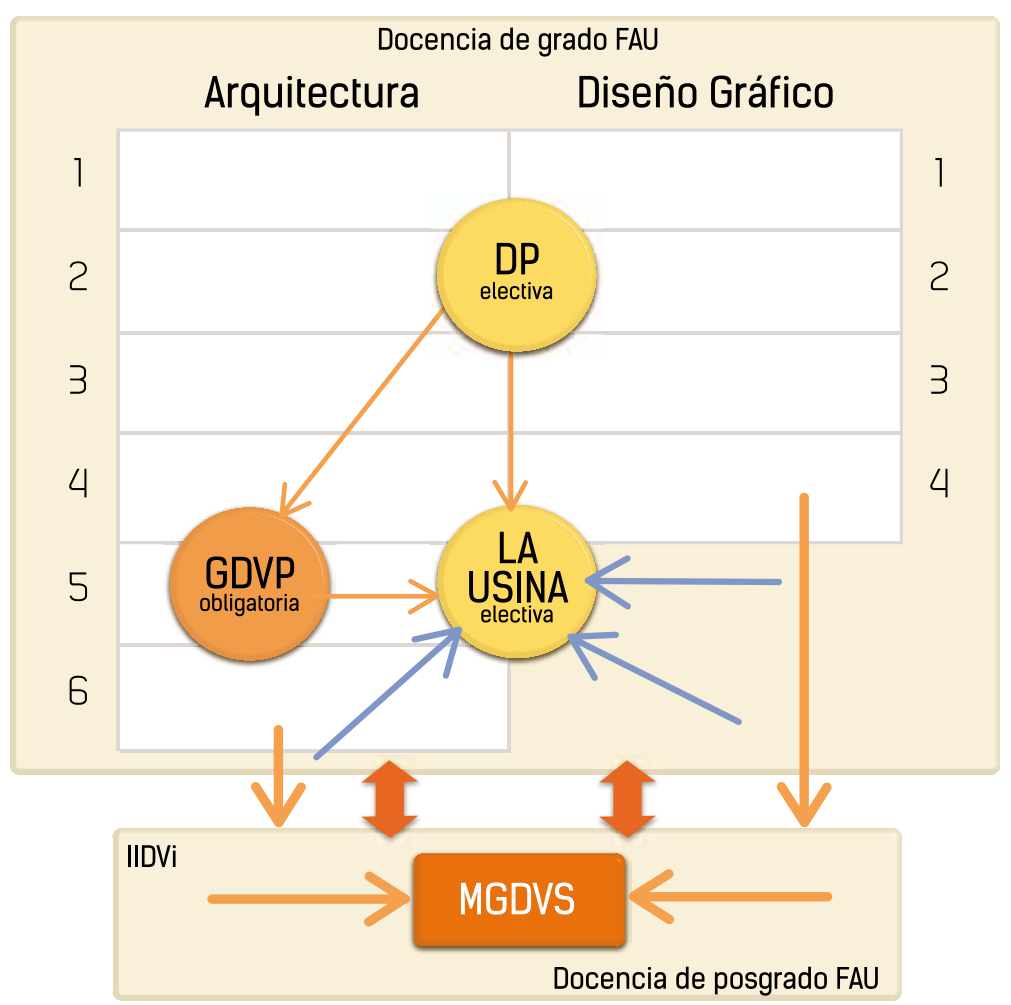

GRADO

GDVP Gestión y Desarrollo de la Vivienda Popular Asignatura obligatoria de Arquitectura $5 .^{\circ}$ año. 1 . $^{\text {er }}$ Cuatrimestre

\section{Diseño Participativo}

DP Asignatura electiva para Arquitectura y Diseño Gráfico (entre $2 .^{\circ}$ y $3 .{ }^{\circ}$ años para ambas carreras. $1 .^{\text {er }}$ cuatrimestre)

LA Práctica Profesional Asistida electiva para Arquitectura

USINA [5. año] y Diseño Gráfico $\left[3 .^{\circ}\right.$ y $4 .^{\circ}$ años] $2 .^{\circ}$ cuatrimestre

Estudiantes de otras carreras
[Economía, Trabajo Social, Abogacia, Ingeniería, otras].
Estudiantes de Arquitectura y Diseño Gráfico.

POSGRADO

$\begin{array}{ll}\text { MGDVS } & \text { Maestría en Gestión y Desar } \\ \text { Dos años de cursada y tesis. }\end{array}$

Articulación institucional del proyecto de docencia de grado con IIDVi. 


\section{REFLEXIONES FINALES}

En síntesis, la evolución de la cátedra -y por ende lo procesual - y la permanente reconfiguración del esquema con que opera emergieron de un proceso que en sí mismo es complejo e implica, en cada uno de sus integrantes, una transformación y un esfuerzo de integración con nuevos actores de campos disciplinares diversos, pero relacionados con el objeto, articulado y retroalimentado en la relación docente-alumno. Además, plantea que la construcción, en tanto modo de acción donde está implicada la intencionalidad, presupone una cierta postura ético-moral, tanto respecto del tratamiento del objeto - que en este caso surge en personas, que se relacionan en el entendimiento y la comprensión, tan importantes para establecer las bases de la comunicación docentealumno-comoparalacomunicación con las personas protagonistas del fenómeno que constituye el objeto de conocimiento. Todas las acciones son pensadas, los significados son permanentemente consensuados entre los docentes y entre docentes y alumnos. El trabajo de la cátedra tiene el sentido de una construcción permanente de conocimiento con calidad científica. Es una "acción", en el sentido de Arendt (Arendt, H., 2009: 200), que ejerce la libertad de trabajar y pensar juntos un mundo mejor. Este agenciamiento (intencionalidad) implica tomar a cargo tanto el proceso como la construcción.

La práctica docente, la extensión y la reflexión constante sobre el accionar pedagógicoconstruyeronunacátedra en transformación permanente, que según las evaluaciones realizadas en forma conjunta con los educandos complementa el aprendizaje disciplinar y aporta a la formación integral del profesional.

\section{BIBLIOGRAFIIA}

BACHELARD, G. (2000). La formación del espíritu científico. Contribución a un psicoanálisis del conocimiento objetivo. Mexico: Siglo XXI Editores. CAMILLONI, A. R. (1997). Los obstáculos epistemológicos en la enseñanza. Barcelona: Gedisa S. A

CARRETERO, M. (1993). Constructivismo y Educación. Capital Federal: Editorial Luis Vives.

FREIRE, P. (2014). Pedagogía del oprimido. (J. Mellado, Trad.) Buenos Aires: Siglo XXI Editores.

GARCíA, R. (2007). Sistemas complejos. Barcelona: Gedisa S. A.

HANNAH, A. (2009). La Condición Humana. Buenos Aires: Paidós. 